\title{
Oscillation criteria for third order nonlinear delay dynamic equations on time scales
}

\author{
by Zhenlai Han (Jinan), Tongxing Li (Jinan), \\ Shurong Sun (Jinan and Rolla, MO) and Fenguuan CaO (Jinan)
}

\begin{abstract}
By means of Riccati transformation technique, we establish some new oscillation criteria for third-order nonlinear delay dynamic equations

$$
\left(\left(x^{\Delta \Delta}(t)\right)^{\gamma}\right)^{\Delta}+p(t) x^{\gamma}(\tau(t))=0
$$

on a time scale $\mathbb{T}$; here $\gamma>0$ is a quotient of odd positive integers and $p$ a real-valued positive rd-continuous function defined on $\mathbb{T}$. Our results not only extend and improve the results of T. S. Hassan [Math. Comput. Modelling 49 (2009)] but also unify the results on oscillation of third-order delay differential equations and third-order delay difference equations.
\end{abstract}

1. Introduction. The study of dynamic equations on time scales was introduced by Hilger [20]. Several authors have expounded on various aspects of this new theory; see the survey paper by Agarwal et al. [1] and the references cited therein. A book on the subject of time scales, by Bohner and Peterson [6], summarizes and organizes much of the time scale calculus; see also their book [7] for advances in dynamic equations on time scales.

In recent years, there has been a great deal of research activity concerning the oscillation and nonoscillation of solutions of various equations on time scales; we refer the reader to the papers [2 -5, 8 11, 15, 18, 21-28, 30-32]. To the best of our knowledge, there seems to be few oscillation results for third-order dynamic equations; see, for example, [12 14, 19, 29]. However, only Hassan's paper [19] deals with third order delay dynamic equations.

In [19], Hassan considered the third-order nonlinear delay dynamic equations

$$
\left(c(t)\left(\left(a(t) x^{\Delta}(t)\right)^{\Delta}\right)^{\gamma}\right)^{\Delta}+f(t, x(\tau(t)))=0, \quad t \in \mathbb{T},
$$

where $\tau(\sigma(t))=\sigma(\tau(t))$ is required, and he established some sufficient conditions for oscillation of (1.1).

2010 Mathematics Subject Classification: 34C10, 34K11, 34N05, 39A21.

Key words and phrases: oscillation, third order, delay dynamic equations, time scales. 
In 2007, Erbe, Peterson and Saker [13] studied third order dynamic equations

$$
x^{\Delta \Delta \Delta}(t)+p(t) x(t)=0, \quad t \in \mathbb{T},
$$

where $p$ is a positive real-valued rd-continuous function on $\mathbb{T}$.

In this paper, we consider third-order nonlinear delay dynamic equations

$$
\left(\left(x^{\Delta \Delta}(t)\right)^{\gamma}\right)^{\Delta}+p(t) x^{\gamma}(\tau(t))=0, \quad t \in \mathbb{T} .
$$

We assume that $\gamma>0$ is a quotient of odd positive integers, the function $p$ is positive, real-valued and rd-continuous, and $\tau: \mathbb{T} \rightarrow \mathbb{T}$ is an rd-continuous function such that $\tau(t) \leq t$ and $\tau(t) \rightarrow \infty(t \rightarrow \infty)$.

Clearly, $(1.2)$ is a special case of (1.3). The purpose of this paper is to establish some new oscillation criteria for (1.3) which guarantee that every solution $x(t)$ of (1.3) oscillates or converges as $t \rightarrow \infty$.

As we are interested in oscillatory behavior, we assume throughout this paper that the given time scale $\mathbb{T}$ is unbounded above. We fix $t_{0} \in \mathbb{T}$; it is convenient to assume $t_{0}>0$. We define the time scale interval $\left[t_{0}, \infty\right)_{\mathbb{T}}$ to be $\left[t_{0}, \infty\right) \cap \mathbb{T}$.

The paper is organized as follows: In Section 2, we establish some new sufficient conditions which guarantee that every solution of (1.3) is oscillatory or converges to a finite number at $\infty$. In Section 3, some examples are considered to illustrate the main results.

2. Main results. In this section we give some new oscillation criteria for (1.3). In order to prove our main results, we will use the formula

$$
\left((x(t))^{\gamma}\right)^{\Delta}=\gamma \int_{0}^{1}\left[h x^{\sigma}(t)+(1-h) x(t)\right]^{\gamma-1} x^{\Delta}(t) d h,
$$

where $x(t)$ is delta differentiable and eventually positive or eventually negative, which is a simple consequence of Keller's chain rule (see Bohner and Peterson [6, Theorem 1.90]).

Throughout this paper, we let

$$
\begin{gathered}
d_{+}(t)=\max \{0, d(t)\}, \quad d_{-}(t)=\max \{0,-d(t)\}, \\
\alpha(t)=\left(\frac{t}{\sigma(t)}\right)^{\gamma}, \quad \gamma \geq 1 ; \quad \alpha(t)=\frac{t}{\sigma(t)}, \quad \gamma<1 .
\end{gathered}
$$

We will need the following auxiliary results.

Lemma 2.1. Assume that $x$ is an eventually positive solution of (1.3). Then for all $t \geq t_{0}$ sufficiently large, either

(i) $x(t)>0, x^{\Delta}(t)>0, x^{\Delta \Delta}(t)>0, x^{\Delta \Delta \Delta}(t)<0$, or

(ii) $x(t)>0, x^{\Delta}(t)<0, x^{\Delta \Delta}(t)>0, x^{\Delta \Delta \Delta}(t)<0$. 
The proof is similar to that of Erbe et al. [12, Lemma 1], so is omitted.

In [6, Section 1.6] the Taylor monomials $\left\{h_{n}(t, s)\right\}_{n=0}^{\infty}$ are defined recursively by

$$
h_{0}(t, s)=1, \quad h_{n+1}(t, s)=\int_{s}^{t} h_{n}(\tau, s) \Delta \tau, \quad t, s \in \mathbb{T}, n \geq 1 .
$$

It follows [6, Section 1.6] that $h_{1}(t, s)=t-s$ for any time scale, but there are no simple general formulas for $n \geq 2$.

Lemma 2.2 ([13, Lemma 4]). Assume that $x$ satisfies case (i) of Lemma 2.1. Then

$$
\liminf _{t \rightarrow \infty} \frac{t x(t)}{h_{2}\left(t, t_{0}\right) x^{\Delta}(t)} \geq 1 .
$$

Lemma 2.3. Assume that $x$ is a solution of (1.3) which satisfies case (i) of Lemma 2.1. If

$$
\int_{t_{0}}^{\infty} p(t)\left(h_{2}\left(\tau(t), t_{0}\right)\right)^{\gamma} \Delta t=\infty
$$

then

$$
x^{\Delta}(t) \geq t x^{\Delta \Delta}(t), \quad x^{\Delta}(t) / t \text { is eventually nonincreasing. }
$$

Proof. Let $x$ be a solution of (1.3) such that case (i) of Lemma 2.1 holds for $t \geq t_{1}$. Define

$$
X(t)=x^{\Delta}(t)-t x^{\Delta \Delta}(t) .
$$

Thus

$$
X^{\Delta}(t)=-\sigma(t) x^{\Delta \Delta \Delta}(t)>0 .
$$

We claim that $X(t)>0$ eventually. Otherwise, there exists $t_{2} \geq t_{1}$ such that $X(t)<0$ for $t \geq t_{2}$. Therefore,

$$
\left(\frac{x^{\Delta}(t)}{t}\right)^{\Delta}=-\frac{X(t)}{t \sigma(t)}>0, \quad t \geq t_{2}
$$

which implies that $x^{\Delta}(t) / t$ is strictly increasing on $\left[t_{2}, \infty\right)_{\mathbb{T}}$. Pick $t_{3} \geq t_{2}$ such that $\tau(t) \geq \tau\left(t_{3}\right) \geq t_{2}$ for $t \geq t_{3}$. Then we have

$$
\frac{x^{\Delta}(\tau(t))}{\tau(t)} \geq \frac{x^{\Delta}\left(\tau\left(t_{3}\right)\right)}{\tau\left(t_{3}\right)}=d>0
$$

so $x^{\Delta}(\tau(t)) \geq d \tau(t)$ for $t \geq t_{3}$. By Lemma 2.2, for any $0<k<1$, there exists $t_{4} \geq t_{3}$ such that

$$
\frac{x(t)}{x^{\Delta}(t)} \geq k \frac{h_{2}\left(t, t_{0}\right)}{t}, \quad t \geq t_{4}
$$


Hence there exists $t_{5} \geq t_{4}$ such that

$$
\begin{aligned}
x(\tau(t)) & \geq k \frac{h_{2}\left(\tau(t), t_{0}\right)}{\tau(t)} x^{\Delta}(\tau(t)) \\
& \geq d k \frac{h_{2}\left(\tau(t), t_{0}\right)}{\tau(t)} \tau(t)=d k h_{2}\left(\tau(t), t_{0}\right), \quad t \geq t_{5} .
\end{aligned}
$$

Integrating both sides of (1.3) from $t_{5}$ to $t$, we have

$$
\left(x^{\Delta \Delta}(t)\right)^{\gamma}-\left(x^{\Delta \Delta}\left(t_{5}\right)\right)^{\gamma}+(d k)^{\gamma} \int_{t_{5}}^{t} p(s)\left(h_{2}\left(\tau(s), t_{0}\right)\right)^{\gamma} \Delta s \leq 0,
$$

which yields

$$
\left(x^{\Delta \Delta}\left(t_{5}\right)\right)^{\gamma} \geq(d k)^{\gamma} \int_{t_{5}}^{t} p(s)\left(h_{2}\left(\tau(s), t_{0}\right)\right)^{\gamma} \Delta s
$$

which contradicts $(2.3)$. Hence, $X(t)>0$ and $x^{\Delta}(t) / t$ is eventually nonincreasing. The proof is complete.

LEMMA 2.4. Assume that $x$ is a solution of (1.3) which satisfies case (ii) of Lemma 2.1. If

$$
\int_{t_{0}}^{\infty} \int_{z}^{\infty}\left[\int_{u}^{\infty} p(s) \Delta s\right]^{1 / \gamma} \Delta u \Delta z=\infty
$$

then $\lim _{t \rightarrow \infty} x(t)=0$.

The proof is similar to that of Erbe et al. [13, Lemma 3], so is omitted.

TheOREM 2.5. Assume that (2.3) holds. Furthermore, assume that there exists a positive function $\delta \in C_{\mathrm{rd}}^{1}\left(\left[t_{0}, \infty\right)_{\mathbb{T}}, \mathbb{R}\right)$ such that for some $0<k<1$,

$$
\limsup _{t \rightarrow \infty} \int_{t_{0}}^{t}\left(k p(s) \delta^{\sigma}(s) C(s)-\frac{1}{(\gamma+1)^{\gamma+1}} \frac{\left(\left(\delta^{\Delta}(s)\right)_{+}\right)^{\gamma+1}}{\alpha^{\gamma}(s) \delta^{\gamma}(\sigma(s))}\right) \Delta s=\infty,
$$

where $C(t)=\left(h_{2}\left(\tau(t), t_{0}\right) / \sigma(t)\right)^{\gamma}$. Then, for every solution $x$ of (1.3), either $x$ is oscillatory or $\lim _{t \rightarrow \infty} x(t)$ exists.

Proof. Suppose that (1.3) has a nonoscillatory solution $x$ on $\left[t_{0}, \infty\right)_{\mathbb{T}}$. We may assume without loss of generality that for some $t_{1} \in\left[t_{0}, \infty\right)_{\mathbb{T}}$ we have $x(t)>0$ and $x(\tau(t))>0$ for all $t \in\left[t_{1}, \infty\right)_{\mathbb{T}}$. Then by Lemma 2.1, $x$ satisfies case (i) or (ii).

Assume case (i) holds. Define the function $\omega$ by

$$
\omega(t)=\delta(t) \frac{\left(x^{\Delta \Delta}(t)\right)^{\gamma}}{\left(x^{\Delta}(t)\right)^{\gamma}}, \quad t \in\left[t_{1}, \infty\right)_{\mathbb{T}} .
$$


Then $\omega(t)>0$. Using the product rule, we have

$$
\omega^{\Delta}(t)=\delta^{\Delta}(t) \frac{\left(x^{\Delta \Delta}(t)\right)^{\gamma}}{\left(x^{\Delta}(t)\right)^{\gamma}}+\delta^{\sigma}(t)\left(\frac{\left(x^{\Delta \Delta}(t)\right)^{\gamma}}{\left(x^{\Delta}(t)\right)^{\gamma}}\right)^{\Delta} .
$$

By the quotient rule, we get

$$
\begin{aligned}
\omega^{\Delta}(t)= & \delta^{\Delta}(t) \frac{\left(x^{\Delta \Delta}(t)\right)^{\gamma}}{\left(x^{\Delta}(t)\right)^{\gamma}} \\
& +\delta^{\sigma}(t) \frac{\left(\left(x^{\Delta \Delta}(t)\right)^{\gamma}\right)^{\Delta}\left(x^{\Delta}(t)\right)^{\gamma}-\left(x^{\Delta \Delta}(t)\right)^{\gamma}\left(\left(x^{\Delta}(t)\right)^{\gamma}\right)^{\Delta}}{\left(x^{\Delta}(t)\right)^{\gamma}\left(x^{\Delta \sigma}(t)\right)^{\gamma}} .
\end{aligned}
$$

From (1.3) and (2.7), we have

$$
\omega^{\Delta}(t)=\frac{\delta^{\Delta}(t)}{\delta(t)} \omega(t)-p(t) \delta^{\sigma}(t) \frac{x^{\gamma}(\tau(t))}{\left(x^{\Delta \sigma}(t)\right)^{\gamma}}-\delta^{\sigma}(t) \frac{\left(x^{\Delta \Delta}(t)\right)^{\gamma}\left(\left(x^{\Delta}(t)\right)^{\gamma}\right)^{\Delta}}{\left(x^{\Delta}(t)\right)^{\gamma}\left(x^{\Delta \sigma}(t)\right)^{\gamma}},
$$

and from (2.2) and (2.4), for any $0<k<1$,

$$
\begin{aligned}
\frac{x^{\gamma}(\tau(t))}{\left(x^{\Delta \sigma}(t)\right)^{\gamma}} & =\frac{x^{\gamma}(\tau(t))}{\left(x^{\Delta}(\tau(t))\right)^{\gamma}} \frac{\left(x^{\Delta}(\tau(t))\right)^{\gamma}}{\left(x^{\Delta \sigma}(t)\right)^{\gamma}} \\
& \geq\left(k^{1 / \gamma} \frac{h_{2}\left(\tau(t), t_{0}\right)}{\tau(t)}\right)^{\gamma}\left(\frac{\tau(t)}{\sigma(t)}\right)^{\gamma}=k\left(\frac{h_{2}\left(\tau(t), t_{0}\right)}{\sigma(t)}\right)^{\gamma} .
\end{aligned}
$$

Hence we obtain

$$
\omega^{\Delta}(t) \leq \frac{\delta^{\Delta}(t)}{\delta(t)} \omega(t)-k p(t) \delta^{\sigma}(t) C(t)-\delta^{\sigma}(t) \frac{\left(x^{\Delta \Delta}(t)\right)^{\gamma}\left(\left(x^{\Delta}(t)\right)^{\gamma}\right)^{\Delta}}{\left(x^{\Delta}(t)\right)^{\gamma}\left(x^{\Delta \sigma}(t)\right)^{\gamma}} .
$$

If $\gamma \geq 1$, from (2.1), we have

$$
\begin{aligned}
\left(\left(x^{\Delta}(t)\right)^{\gamma}\right)^{\Delta} & =\gamma \int_{0}^{1}\left[h x^{\Delta \sigma}(t)+(1-h) x^{\Delta}(t)\right]^{\gamma-1} x^{\Delta \Delta}(t) d h \\
& \geq \gamma\left(x^{\Delta}(t)\right)^{\gamma-1} x^{\Delta \Delta}(t) .
\end{aligned}
$$

By (2.8),

$$
\omega^{\Delta}(t) \leq \frac{\delta^{\Delta}(t)}{\delta(t)} \omega(t)-k p(t) \delta^{\sigma}(t) C(t)-\gamma \delta^{\sigma}(t) \frac{\left(x^{\Delta \Delta}(t)\right)^{\gamma+1}}{x^{\Delta}(t)\left(x^{\Delta \sigma}(t)\right)^{\gamma}} .
$$

Since $x^{\Delta}(t) / t$ is nonincreasing, we have $x^{\Delta}(t) / t \geq x^{\Delta \sigma}(t) / \sigma(t)$, so

$$
\omega^{\Delta}(t) \leq \frac{\delta^{\Delta}(t)}{\delta(t)} \omega(t)-k p(t) \delta^{\sigma}(t) C(t)-\gamma\left(\frac{t}{\sigma(t)}\right)^{\gamma} \delta^{\sigma}(t) \frac{\left(x^{\Delta \Delta}(t)\right)^{\gamma+1}}{\left(x^{\Delta}(t)\right)^{\gamma+1}} .
$$

By (2.7), we obtain

$$
\omega^{\Delta}(t) \leq \frac{\left(\delta^{\Delta}(t)\right)_{+}}{\delta(t)} \omega(t)-k p(t) \delta^{\sigma}(t) C(t)-\gamma\left(\frac{t}{\sigma(t)}\right)^{\gamma} \frac{\delta^{\sigma}(t)}{\delta^{\lambda}(t)} \omega^{\lambda}(t),
$$


where $\lambda=(\gamma+1) / \gamma$. If $\gamma<1$, from (2.1), we have

$$
\begin{aligned}
\left(\left(x^{\Delta}(t)\right)^{\gamma}\right)^{\Delta} & =\gamma \int_{0}^{1}\left[h x^{\Delta \sigma}(t)+(1-h) x^{\Delta}(t)\right]^{\gamma-1} x^{\Delta \Delta}(t) d h \\
& \geq \gamma\left(x^{\Delta \sigma}(t)\right)^{\gamma-1} x^{\Delta \Delta}(t) .
\end{aligned}
$$

By $(2.8)$,

$$
\begin{aligned}
\omega^{\Delta}(t) & \leq \frac{\delta^{\Delta}(t)}{\delta(t)} \omega(t)-k p(t) \delta^{\sigma}(t) C(t)-\gamma \delta^{\sigma}(t) \frac{\left(x^{\Delta \Delta}(t)\right)^{\gamma+1}}{\left(x^{\Delta}(t)\right)^{\gamma} x^{\Delta \sigma}(t)} \\
& \leq \frac{\delta^{\Delta}(t)}{\delta(t)} \omega(t)-k p(t) \delta^{\sigma}(t) C(t)-\gamma \frac{t}{\sigma(t)} \delta^{\sigma}(t) \frac{\left(x^{\Delta \Delta}(t)\right)^{\gamma+1}}{\left(x^{\Delta}(t)\right)^{\gamma+1}} .
\end{aligned}
$$

By (2.7), we obtain

$$
\omega^{\Delta}(t) \leq \frac{\left(\delta^{\Delta}(t)\right)_{+}}{\delta(t)} \omega(t)-k p(t) \delta^{\sigma}(t) C(t)-\gamma \frac{t}{\sigma(t)} \frac{\delta^{\sigma}(t)}{\delta^{\lambda}(t)} \omega^{\lambda}(t) .
$$

By (2.9), (2.10) and the definition of $\alpha(t)$, we have, as $\gamma>0$,

$$
\omega^{\Delta}(t) \leq \frac{\left(\delta^{\Delta}(t)\right)_{+}}{\delta(t)} \omega(t)-k p(t) \delta^{\sigma}(t) C(t)-\gamma \alpha(t) \frac{\delta^{\sigma}(t)}{\delta^{\lambda}(t)} \omega^{\lambda}(t) .
$$

Set

$$
A=\left[\gamma \alpha(t) \frac{\delta^{\sigma}(t)}{\delta^{\lambda}(t)}\right]^{1 / \lambda} \omega(t), \quad B=\left[\frac{\left(\delta^{\Delta}(t)\right)_{+}}{\lambda \delta(t)}\left(\gamma \alpha(t) \frac{\delta^{\sigma}(t)}{\delta^{\lambda}(t)}\right)^{-1 / \lambda}\right]^{1 /(\lambda-1)} .
$$

Using the inequality

$$
\lambda A B^{\lambda-1}-A^{\lambda} \leq(\lambda-1) B^{\lambda}, \quad \lambda \geq 1,
$$

we have

$$
\frac{\left(\delta^{\Delta}(t)\right)_{+}}{\delta(t)} \omega(t)-\gamma \alpha(t) \frac{\delta^{\sigma}(t)}{\delta^{\lambda}(t)} \omega^{\lambda}(t) \leq \frac{1}{(\gamma+1)^{\gamma+1}} \frac{\left(\left(\delta^{\Delta}(t)\right)_{+}\right)^{\gamma+1}}{\alpha^{\gamma}(t) \delta^{\gamma}(\sigma(t))},
$$

hence from (2.11), we get

$$
\omega^{\Delta}(t) \leq-k p(t) \delta^{\sigma}(t) C(t)+\frac{1}{(\gamma+1)^{\gamma+1}} \frac{\left(\left(\delta^{\Delta}(t)\right)_{+}\right)^{\gamma+1}}{\alpha^{\gamma}(t) \delta^{\gamma}(\sigma(t))} .
$$

Integrating the inequality (2.12) from $t_{1}$ to $t$, we obtain

$$
\begin{aligned}
-\omega\left(t_{1}\right) & \leq \omega(t)-\omega\left(t_{1}\right) \\
& \leq-\int_{t_{1}}^{t}\left(k p(s) \delta^{\sigma}(s) C(s)-\frac{1}{(\gamma+1)^{\gamma+1}} \frac{\left(\left(\delta^{\Delta}(s)\right)_{+}\right)^{\gamma+1}}{\alpha^{\gamma}(s) \delta^{\gamma}(\sigma(s))}\right) \Delta s,
\end{aligned}
$$

which yields

$$
\int_{t_{1}}^{t}\left(k p(s) \delta^{\sigma}(s) C(s)-\frac{1}{(\gamma+1)^{\gamma+1}} \frac{\left(\left(\delta^{\Delta}(s)\right)_{+}\right)^{\gamma+1}}{\alpha^{\gamma}(s) \delta^{\gamma}(\sigma(s))}\right) \Delta s \leq \omega\left(t_{1}\right)
$$


for all large $t$, which contradicts (2.6). If case (ii) holds, then $\lim _{t \rightarrow \infty} x(t)$ exists. The proof is complete.

REMARK 2.6. From Theorem 2.5, we can obtain different conditions for oscillation of all solutions of (1.3) with different choices of $\delta$.

For example, let $\gamma \geq 1, \delta(t)=t^{\gamma}$. Then $\left(t^{\gamma}\right)^{\Delta} \leq \gamma(\sigma(t))^{\gamma-1}$. Now Theorem 2.5 yields the following result.

Corollary 2.7. Assume that (2.3) holds and $\gamma \geq 1$. If for some $0<$ $k<1$,

(2.13) $\limsup _{t \rightarrow \infty} \int_{t_{0}}^{t}\left(k p(s)\left(h_{2}\left(\tau(s), t_{0}\right)\right)^{\gamma}-\left(\frac{\gamma}{\gamma+1}\right)^{\gamma+1} \frac{(\sigma(s))^{\gamma^{2}-1}}{s^{\gamma^{2}}}\right) \Delta s=\infty$, then for every solution $x$ of (1.3), either $x$ is oscillatory or $\lim _{t \rightarrow \infty} x(t)$ exists.

The following theorem gives a Kamenev-type oscillation criterion for (1.3).

Theorem 2.8. Assume (2.3) holds. Let $\delta, C$ be as in Theorem 2.5. If for some $0<k<1$ and $m \geq 1$,

$\limsup _{t \rightarrow \infty} \frac{1}{t^{m}} \int_{t_{0}}^{t}(t-s)^{m}\left(k p(s) \delta^{\sigma}(s) C(s)-\frac{1}{(\gamma+1)^{\gamma+1}} \frac{\left(\left(\delta^{\Delta}(s)\right)_{+}\right)^{\gamma+1}}{\alpha^{\gamma}(s) \delta^{\gamma}(\sigma(s))}\right) \Delta s=\infty$, then for every solution $x$ of (1.3), either $x$ is oscillatory or $\lim _{t \rightarrow \infty} x(t)$ exists.

Proof. Suppose that (1.3) has a nonoscillatory solution $x$ on $\left[t_{0}, \infty\right)_{\mathbb{T}}$. We may assume that for some $t_{1} \in\left[t_{0}, \infty\right)_{\mathbb{T}}$ we have $x(t)>0$ and $x(\tau(t))>0$ for all $t \in\left[t_{1}, \infty\right)_{\mathbb{T}}$. Then by Lemma $2.1, x$ satisfies case (i) or (ii).

Assume case (i) holds. Define the function $\omega$ as in (2.7). We proceed as in the proof of Theorem 2.5 to get (2.12). Then from (2.12), we have

$$
k p(t) \delta^{\sigma}(t) C(t)-\frac{1}{(\gamma+1)^{\gamma+1}} \frac{\left(\left(\delta^{\Delta}(t)\right)_{+}\right)^{\gamma+1}}{\alpha^{\gamma}(t) \delta^{\gamma}(\sigma(t))} \leq-\omega^{\Delta}(t) .
$$

Therefore,

$$
\begin{aligned}
\int_{t_{1}}^{t}(t-s)^{m}\left[k p(s) \delta^{\sigma}(s) C(s)-\frac{1}{(\gamma+1)^{\gamma+1}}\right. & \left.\frac{\left(\left(\delta^{\Delta}(s)\right)_{+}\right)^{\gamma+1}}{\alpha^{\gamma}(s) \delta^{\gamma}(\sigma(s))}\right] \Delta s \\
& \leq-\int_{t_{1}}^{t}(t-s)^{m} \omega^{\Delta}(s) \Delta s .
\end{aligned}
$$


By integration by parts, the right hand side equals

$$
\int_{t_{1}}^{t}(t-s)^{m} \omega^{\Delta}(s) \Delta s=\left.(t-s)^{m} \omega(s)\right|_{t_{1}} ^{t}-\int_{t_{1}}^{t}\left((t-s)^{m}\right)^{\Delta_{s}} \omega(\sigma(s)) \Delta s .
$$

Note that since $\left((t-s)^{m}\right)^{\Delta_{s}} \leq-m(t-\sigma(s))^{m-1} \leq 0$ for $t \geq \sigma(s), m \geq 1$ (see Erbe et al. [14]), from (2.15) we have

$$
\int_{t_{1}}^{t}(t-s)^{m}\left[k p(s) \delta^{\sigma}(s) C(s)-\frac{1}{(\gamma+1)^{\gamma+1}} \frac{\left(\left(\delta^{\Delta}(s)\right)_{+}\right)^{\gamma+1}}{\alpha^{\gamma}(s) \delta^{\gamma}(\sigma(s))}\right] \Delta s \leq\left(t-t_{1}\right)^{m} \omega\left(t_{1}\right) \text {. }
$$

Then

$$
\begin{aligned}
\frac{1}{t^{m}} \int_{t_{1}}^{t}(t-s)^{m}\left[k p(s) \delta^{\sigma}(s) C(s)-\frac{1}{(\gamma+1)^{\gamma+1}} \frac{\left(\left(\delta^{\Delta}(s)\right)_{+}\right)^{\gamma+1}}{\alpha^{\gamma}(s) \delta^{\gamma}(\sigma(s))}\right] \Delta s & \\
& \leq\left(\frac{t-t_{1}}{t}\right)^{m} \omega\left(t_{1}\right),
\end{aligned}
$$

which contradicts 2.14). If case (ii) holds, then $\lim _{t \rightarrow \infty} x(t)$ exists. The proof is complete.

The following theorem gives a Philos-type oscillation criterion for (1.3).

Theorem 2.9. Assume that (2.3) holds. Let $\delta, C$ be as in Theorem 2.5. Furthermore, assume that there exist functions $H, h \in C_{\mathrm{rd}}(\mathbb{D}, \mathbb{R})$, where $\mathbb{D} \equiv\left\{(t, s): t \geq s \geq t_{0}\right\}$ such that

$$
H(t, t)=0, \quad t \geq t_{0}, \quad H(t, s)>0, \quad t>s \geq t_{0},
$$

and $H$ has a nonpositive continuous $\Delta$-partial derivative $H^{\Delta_{s}}(t, s)$ with respect to the second variable which satisfies

$$
H^{\Delta_{s}}(\sigma(t), s)+H(\sigma(t), \sigma(s)) \frac{\delta^{\Delta}(s)}{\delta(s)}=-\frac{h(t, s)}{\delta(s)}(H(\sigma(t), \sigma(s)))^{\gamma /(\gamma+1)} .
$$

If for some $0<k<1$,

$$
\limsup _{t \rightarrow \infty} \frac{1}{H\left(\sigma(t), t_{0}\right)} \int_{t_{0}}^{\sigma(t)} K(t, s) \Delta s=\infty
$$

where

$$
K(t, s)=k H(\sigma(t), \sigma(s)) p(s) \delta^{\sigma}(s) C(s)-\frac{1}{(\gamma+1)^{\gamma+1}} \frac{\left(h_{-}(t, s)\right)^{\gamma+1}}{\alpha^{\gamma}(s) \delta^{\gamma}(\sigma(s))},
$$

then for every solution $x$ of (1.3), either $x$ is oscillatory or $\lim _{t \rightarrow \infty} x(t)$ exists.

The proof is similar to that of Theorem 2.5 in Han et al. [16] using the inequality (2.11). We omit the details. 
In the following theorem we establish new oscillation criteria for (1.3) which are different from those above.

Theorem 2.10. Assume that (2.3) holds. If for some $0<k<1$,

$$
\limsup _{t \rightarrow \infty} \int_{\tau(t)}^{t} p(s)\left(h_{2}\left(\tau(s), t_{0}\right)\right)^{\gamma} \Delta s>\frac{1}{k},
$$

then for every solution $x$ of (1.3), either $x$ is oscillatory or $\lim _{t \rightarrow \infty} x(t)$ exists.

Proof. Suppose that (1.3) has a nonoscillatory solution $x$ on $\left[t_{0}, \infty\right)_{\mathbb{T}}$. We may assume that for some $t_{1} \in\left[t_{0}, \infty\right)_{\mathbb{T}}$ we have $x(t)>0$ and $x(\tau(t))>0$ for all $t \in\left[t_{1}, \infty\right)_{\mathbb{T}}$. Then by Lemma 2.1, $x$ satisfies case (i) or (ii).

Assume case (i) holds. From (2.2) and (2.4), for any $0<k<1$, we have

$$
\begin{aligned}
x(\tau(t)) & \geq k^{1 / \gamma} \frac{h_{2}\left(\tau(t), t_{0}\right)}{\tau(t)} x^{\Delta}(\tau(t)) \geq k^{1 / \gamma} \frac{h_{2}\left(\tau(t), t_{0}\right)}{\tau(t)} \tau(t) x^{\Delta \Delta}(\tau(t)) \\
& =k^{1 / \gamma} h_{2}\left(\tau(t), t_{0}\right) x^{\Delta \Delta}(\tau(t)) .
\end{aligned}
$$

By (1.3), we obtain

$$
\left(\left(x^{\Delta \Delta}(t)\right)^{\gamma}\right)^{\Delta}+k p(t)\left(h_{2}\left(\tau(t), t_{0}\right)\right)^{\gamma}\left(x^{\Delta \Delta}(\tau(t))\right)^{\gamma} \leq 0 .
$$

Set $z(t)=\left(x^{\Delta \Delta}(t)\right)^{\gamma}$. Then

$$
z^{\Delta}(t)+k p(t)\left(h_{2}\left(\tau(t), t_{0}\right)\right)^{\gamma} z(\tau(t)) \leq 0 .
$$

Integrating the above inequality from $\tau(t)$ to $t$, we have

$$
\begin{aligned}
0 & \geq \int_{\tau(t)}^{t} z^{\Delta}(s) \Delta s+\int_{\tau(t)}^{t} k p(s)\left(h_{2}\left(\tau(s), t_{0}\right)\right)^{\gamma} z(\tau(s)) \Delta s \\
& =z(t)-z(\tau(t))+\int_{\tau(t)}^{t} k p(s)\left(h_{2}\left(\tau(s), t_{0}\right)\right)^{\gamma} z(\tau(s)) \Delta s \\
& \geq z(t)-z(\tau(t))+z(\tau(t)) \int_{\tau(t)}^{t} k p(s)\left(h_{2}\left(\tau(s), t_{0}\right)\right)^{\gamma} \Delta s \\
& =z(t)+z(\tau(t))\left[\int_{\tau(t)}^{t} k p(s)\left(h_{2}\left(\tau(s), t_{0}\right)\right)^{\gamma} \Delta s-1\right]>0
\end{aligned}
$$

by (2.17), a contradiction. If case (ii) holds, then $\lim _{t \rightarrow \infty} x(t)$ exists. The proof is complete.

REMARK 2.11. Note that Theorem 2.10 is not applicable to equations of type (1.3) with $\tau(t)=t$. So the delay appearing in (1.3) plays a crucial role in the qualitative behavior. 
Theorem 2.12. Assume that (2.3) holds. If

$$
\limsup _{t \rightarrow \infty} t^{\gamma} \int_{t}^{\infty} p(s)\left(\frac{h_{2}\left(\tau(s), t_{0}\right)}{s}\right)^{\gamma} \Delta s=\infty
$$

then for every solution $x$ of (1.3), either $x$ is oscillatory or $\lim _{t \rightarrow \infty} x(t)$ exists.

Proof. Suppose that (1.3) has a nonoscillatory solution $x$ on $\left[t_{0}, \infty\right)_{\mathbb{T}}$. We may assume that for some $t_{1} \in\left[t_{0}, \infty\right)_{\mathbb{T}}$ we have $x(t)>0$ and $x(\tau(t))>0$ for all $t \in\left[t_{1}, \infty\right)_{\mathbb{T}}$. Then by Lemma $2.1, x$ satisfies case (i) or (ii).

Assume case (i) holds. Set $y(t)=\left(x^{\Delta \Delta}(t)\right)^{\gamma}$. By (1.3), for $T \geq t \geq t_{1}$,

$$
y(T)=y(t)+\int_{t}^{T} y^{\Delta}(s) \Delta s=y(t)-\int_{t}^{T} p(s) x^{\gamma}(\tau(s)) \Delta s,
$$

and hence

$$
\int_{t}^{T} p(s) x^{\gamma}(\tau(s)) \Delta s=y(t)-y(T) \leq y(t)=\left(x^{\Delta \Delta}(t)\right)^{\gamma} .
$$

By (2.2) and (2.4), for any $0<k<1$,

$$
\begin{aligned}
\left(x^{\Delta}(t)\right)^{\gamma} & \geq t^{\gamma}\left(x^{\Delta \Delta}(t)\right)^{\gamma} \geq t^{\gamma} \int_{t}^{\infty} p(s) x^{\gamma}(\tau(s)) \Delta s \\
& \geq t^{\gamma} \int_{t}^{\infty} k p(s)\left(\frac{h_{2}\left(\tau(s), t_{0}\right)}{\tau(s)} x^{\Delta}(\tau(s))\right)^{\gamma} \Delta s \\
& \geq k t^{\gamma} \int_{t}^{\infty} p(s)\left(\frac{h_{2}\left(\tau(s), t_{0}\right)}{\tau(s)} \frac{\tau(s)}{s} x^{\Delta}(s)\right)^{\gamma} \Delta s \\
& =k t^{\gamma} \int_{t}^{\infty} p(s)\left(\frac{h_{2}\left(\tau(s), t_{0}\right)}{s}\right)^{\gamma}\left(x^{\Delta}(s)\right)^{\gamma} \Delta s \\
& \geq k t^{\gamma}\left(x^{\Delta}(t)\right)^{\gamma} \int_{t}^{\infty} p(s)\left(\frac{h_{2}\left(\tau(s), t_{0}\right)}{s}\right)^{\gamma} \Delta s,
\end{aligned}
$$

which yields

$$
t^{\gamma} \int_{t}^{\infty} p(s)\left(\frac{h_{2}\left(\tau(s), t_{0}\right)}{s}\right)^{\gamma} \Delta s \leq \frac{1}{k}
$$

contrary to (2.18). If case (ii) holds, then $\lim _{t \rightarrow \infty} x(t)$ exists. The proof is complete.

From Lemma 2.4, we have the following result.

Theorem 2.13. Assume that (2.3) and (2.5) hold. Let $\delta, C$ be as in Theorem 2.5, and $0<k<1$. If one of the conditions (2.6), (2.14), (2.16), 
(2.17) or (2.18) holds, then every solution of (1.3) oscillates or converges to zero.

REMARK 2.14. If we define the Riccati substitution as (2.7), then we can obtain

$$
\omega^{\Delta}(t)=\left(x^{\Delta \Delta \sigma}(t)\right)^{\gamma}\left(\frac{\delta(t)}{\left(x^{\Delta}(t)\right)^{\gamma}}\right)^{\Delta}+\frac{\delta(t)}{\left(x^{\Delta}(t)\right)^{\gamma}}\left(\left(x^{\Delta \Delta}(t)\right)^{\gamma}\right)^{\Delta} .
$$

From this formula, we can establish some oscillation criteria which are different from the above results; we leave this to the interested reader.

3. Examples. The following examples illustrate our main results.

EXAMPLE 3.1. Consider the equation

$$
\left(\left(x^{\Delta \Delta}(t)\right)^{\gamma}\right)^{\Delta}+\frac{\beta}{t\left(h_{2}\left(\tau(t), t_{0}\right)\right)^{\gamma}} x^{\gamma}(\tau(t))=0, \quad t \in\left[t_{0}, \infty\right)_{\mathbb{T}},
$$

where $\beta>0, \gamma \geq 1$ is a quotient of odd positive integers, and $\sigma(t) \leq \alpha t$ for $\alpha \geq 1$.

It is easy to see that (2.3) holds. Let

$$
p(t)=\frac{\beta}{t\left(h_{2}\left(\tau(t), t_{0}\right)\right)^{\gamma}} .
$$

From (2.11), we have

$$
\begin{aligned}
& \limsup _{t \rightarrow \infty} \int_{t_{0}}^{t}\left(k p(s)\left(h_{2}\left(\tau(s), t_{0}\right)\right)^{\gamma}-\left(\frac{\gamma}{\gamma+1}\right)^{\gamma+1} \frac{(\sigma(s))^{\gamma^{2}-1}}{s^{\gamma^{2}}}\right) \Delta s \\
& \geq\left(k \beta-\left(\frac{\gamma}{\gamma+1}\right)^{\gamma+1} \alpha^{\gamma^{2}-1}\right) \limsup _{t \rightarrow \infty} \int_{t_{0}}^{t} \frac{\Delta s}{s} .
\end{aligned}
$$

Hence if $\beta>(\gamma /(\gamma+1))^{\gamma+1} \alpha^{\gamma^{2}-1} / k$ for some $0<k<1$, then (2.13) holds, so by Corollary 2.7, every solution $x$ of (3.1) is oscillatory or convergent.

Example 3.2. Consider the equation

$$
x^{\Delta \Delta \Delta}(t)+\frac{\beta}{t \tau^{2}(t)} x(\tau(t))=0, \quad t \in[1, \infty)_{\mathbb{T}},
$$

where $\mathbb{T}=q^{\mathbb{N}_{0}}, q>1, \beta>0$. Here we have $\sigma(t)=q t, h_{2}\left(\tau(t), t_{0}\right)=$ $h_{2}(\tau(t), 1)=(\tau(t)-1)(\tau(t)-q) /(1+q)$. Let $p(t)=\beta /\left(t \tau^{2}(t)\right), \gamma=1$. Then

$$
\begin{aligned}
\int_{t_{0}}^{\infty} p(t)\left(h_{2}\left(\tau(t), t_{0}\right)\right)^{\gamma} \Delta t & =\int_{1}^{\infty} \frac{\beta}{t \tau^{2}(t)} \frac{(\tau(t)-1)(\tau(t)-q)}{1+q} \Delta t \\
& \geq \frac{\alpha \beta}{1+q} \int_{1}^{\infty} \frac{\Delta t}{t}=\infty \quad \text { for some } 0<\alpha<1,
\end{aligned}
$$


so (2.3) holds. It is easy to see that $(2.5)$ holds. Let $\delta(t)=t$. Moreover,

$$
\begin{aligned}
\limsup _{t \rightarrow \infty} \int_{t_{0}}^{t}\left(k p(s) \delta^{\sigma}(s) C\right. & \left.(s)-\frac{1}{(\gamma+1)^{\gamma+1}} \frac{(\sigma(s))^{\gamma^{2}}\left(\left(\delta^{\Delta}(s)\right)_{+}\right)^{\gamma+1}}{s^{\gamma^{2}} \delta^{\gamma}(\sigma(s))}\right) \Delta s \\
& =\limsup _{t \rightarrow \infty} \int_{1}^{t}\left(k \frac{\beta}{s \tau^{2}(s)} \frac{(\tau(s)-1)(\tau(s)-q)}{1+q}-\frac{1}{4 s}\right) \Delta s \\
\geq & \left(\frac{k \alpha \beta}{1+q}-\frac{1}{4}\right) \limsup _{t \rightarrow \infty} \int_{1}^{t} \frac{\Delta s}{s} .
\end{aligned}
$$

Hence if $\beta>(1+q) /(4 k \alpha)$ for some $0<k<1$ and $0<\alpha<1$, then (2.6) holds, so by Theorem 2.13, every soluion $x$ of (3.2) is either oscillatory or converges to zero.

REMARK 3.1. Note that the results due to Hassan [19] do not apply to equations (3.1) and (3.2) since $\tau(\sigma(t)) \not \equiv \sigma(\tau(t))$. Hence our results are new.

Acknowledgements. The authors sincerely thank the reviewers for their valuable suggestions and useful comments that have led to the present improved version of the original manuscript.

This research is supported by the Natural Science Foundation of China (60774004, 60904024), China Postdoctoral Science Foundation Funded Project (20080441126, 200902564), Shandong Postdoctoral Funded Project (200802018), Shandong Research Funds (Y2008A28, ZR2009AL003), and University of Jinan Research Funds for Doctors (B0621, XBS0843).

\section{References}

[1] R. P. Agarwal, M. Bohner, D. O'Regan and A. Peterson, Dynamic equations on time scales: a survey, J. Comput. Appl. Math. 141 (2002), 1-26.

[2] R. P. Agarwal, M. Bohner and S. H. Saker, Oscillation of second order delay dynamic equations, Canad. Appl. Math. Quart. 13 (2005), 1-18.

[3] E. Akin-Bohner, M. Bohner and S. H. Saker, Oscillation criteria for a certain class of second order Emden-Fowler dynamic equations, Electron. Trans. Numer. Anal. 27 (2007), 1-12.

[4] E. Akin-Bohner and J. Hoffacker, Oscillation properties of an Emden-Fowler type equation on discrete time scales, J. Difference Equations Appl. 9 (2003), 603-612.

[5] M. Bohner, Some oscillation criteria for first order delay dynamic equations, Far East J. Appl. Math. 18 (2005), 289-304.

[6] M. Bohner and A. Peterson, Dynamic Equations on Time Scales: An Introduction with Applications, Birkhäuser, Boston, 2001.

[7] —, 一, Advances in Dynamic Equations on Time Scales, Birkhäuser, Boston, 2003.

[8] M. Bohner and S. H. Saker, Oscillation of second order nonlinear dynamic equations on time scales, Rocky Mountain J. Math. 34 (2004), 1239-1254. 
[9] L. Erbe and A. Peterson, Positive solutions for a nonlinear differential equation on a measure chain, Math. Comput. Modelling 32 (2000), 571-585.

[10] L. Erbe, A. Peterson and S. H. Saker, Oscillation criteria for second-order nonlinear dynamic equations on time scales, J. London Math. Soc. 76 (2003), 701-714.

[11] - - - - Oscillation criteria for second-order nonlinear delay dynamic equations, J. Math. Anal. Appl. 333 (2007), 505-522.

[12] - - - - Asymptotic behavior of solutions of a third-order nonlinear dynamic equation on time scales, J. Comput. Appl. Math. 181 (2005), 92-102.

[13] - - - - , Hille and Nehari type criteria for third order dynamic equations, J. Math. Anal. Appl. 329 (2007), 112-131.

[14] -, -, -, Oscillation and asymptotic behavior of a third-order nonlinear dynamic equation, Canad. Appl. Math. Quart. 14 (2006), 129-147.

[15] S. R. Grace, R. P. Agarwal, M. Bohner and D. O'Regan, Oscillation of second-order strongly superlinear and strongly sublinear dynamic equations, Comm. Nonlinear Sci. Numer. Simulation 14 (2009), 3463-3471.

[16] Z. Han, T. Li, S. Sun and C. Zhang, Oscillation for second-order nonlinear delay dynamic equations on time scales, Adv. Difference Equations 2009, art. ID 756171, $13 \mathrm{pp}$.

[17] Z. Han, S. Sun and B. Shi, Oscillation criteria for a class of second order EmdenFowler delay dynamic equations on time scales, J. Math. Anal. Appl. 334 (2007), 847-858.

[18] T. S. Hassan, Oscillation criteria for half-linear dynamic equations on time scales, J. Math. Anal. Appl. 345 (2008), 176-185.

[19] - Oscillation of third order nonlinear delay dynamic equations on time scales, Math. Comput. Modelling 49 (2009), 1573-1586.

[20] S. Hilger, Analysis on measure chains - a unified approach to continuous and discrete calculus, Results Math. 18 (1990), 18-56.

[21] B. Jia, L. Erbe and A. Peterson, New comparison and oscillation theorems for second-order half-linear dynamic equations on time scales, Comput. Math. Appl. 56 (2008), 2744-2756.

[22] Y. Sahiner, Oscillation of second-order delay differential equations on time scales, Nonlinear Anal. 63 (2005), 1073-1080.

[23] S. H. Saker, Oscillation criteria of second-order half-linear dynamic equations on time scales, J. Comput. Appl. Math. 177 (2005), 375-387.

[24] -, Oscillation of nonlinear dynamic equations on time scales, Appl. Math. Comput. 148 (2004), 81-91.

[25] —, New oscillation criteria for second-order nonlinear dynamic equations on time scales, Nonlinear Funct. Anal. Appl. 11 (2006), 351-370.

[26] S. H. Saker, R. P. Agarwal and D. O'Regan, Oscillation results for second-order nonlinear neutral delay dynamic equations on time scales, Appl. Anal. 86 (2007), $1-17$.

[27] S. Sun, Z. Han and C. Zhang, Oscillation of second-order delay dynamic equations on time scales, J. Appl. Math. Comput. 30 (2009), 459-468.

[28] - - - - -, Oscillation criteria of second-order Emden-Fowler neutral delay dynamic equations on time scales, J. Shanghai Jiaotong Univ. 42 (2008), 2070-2075.

[29] Z. Yu and Q. Wang, Asymptotic behavior of solutions of third-order nonlinear dynamic equations on time scales, J. Comput. Appl. Math. 225 (2009), 531-540.

[30] B. G. Zhang and X. Deng, Oscillation of delay differential equations on time scales, Math. Comput. Modelling 36 (2002), 1307-1318. 
[31] B. G. Zhang and S. Zhu, Oscillation of second order nonlinear delay dynamic equations on time scales, Comput. Math. Appl. 49 (2005), 599-609.

[32] Z. Zhu and Q. Wang, Existence of nonoscillatory solutions to neutral dynamic equations on time scales, J. Math. Anal. Appl. 335 (2007), 751-762.

Zhenlai Han (corresponding author)

Tongxing Li

School of Science

University of Jinan

Jinan, Shandong 250022, P.R. China

and

School of Control Science and Engineering

Shandong University

Jinan, Shandong 250061, P.R. China

E-mail: hanzhenlai@163.com

Shurong Sun

School of Science

University of Jinan

Jinan, Shandong 250022, P.R. China

and

Department of Mathematics and Statistics

Missouri University of Science and Technology

Rolla, MO 65409-0020, U.S.A.

E-mail: sshrong@163.com

Received 3.9.2009

and in final form 3.12.2009 